\title{
Factors Associated with Unplanned Reinterventions and Their Relation to Early Mortality
}

\section{after Pediatric Cardiac Surgery}

Dan M. Dorobantu, MD, ${ }^{1,2}$ Deborah Ridout, MSc, ${ }^{3}$ Katherine L. Brown, MD, MPH, ${ }^{4}$ Warren

Rodrigues, MRCP, ${ }^{4}$ Mansour TA. Sharabiani, $\mathrm{PhD},{ }^{5}$ Christina Pagel, $\mathrm{PhD},{ }^{6}$ David Anderson, FRCS, ${ }^{7}$ Paul Wellman, BSc, ${ }^{7}$ Andrew McLean, FRCS, ${ }^{8}$ Jane Cassidy, MRCP, ${ }^{9}$ David J. Barron, MD,,${ }^{9,10}$ Victor T. Tsang, FRCS, ${ }^{4}$ Serban C. Stoica, FRCS,, 11

1. Faculty of Health Sciences, University of Bristol, Bristol, United Kingdom

2. Children's Health and Exercise Research Centre, University of Exeter, Exeter, United Kingdom

3. Population, Policy and Practice Programme, University College London, Great Ormond Street Institute of Child Health, London, United Kingdom

4. Cardiac and Critical Care Division, Great Ormond Street Hospital, London, United Kingdom

5. Department of Primary Care \& Public Health, School of Public Health, Imperial College of London, London, United Kingdom

6. Clinical Operational Research Unit, University College London, London, United Kingdom

7. Departments of Paediatric Cardiology, Intensive Care and Cardiac Surgery, Evelina London Children's Hospital, London, United Kingdom

8. Department of Paediatric Cardiac Surgery, Royal Hospital for Children Glasgow, Glasgow, United Kingdom

9. Department of Intensive Care and Paediatric Cardiac Surgery, Birmingham Children's Hospital, Birmingham, United Kingdom

10. Division of Cardiovascular Surgery, Toronto Hospital for Sick Children, Toronto, ON, Canada

11. Department of Paediatric Cardiac Surgery, Bristol Royal Hospital for Children, Bristol, United Kingdom

Presented at the 2020 American Association of Thoracic Surgery Meeting 
Corresponding author: Mr. Serban Stoica, email: Serban.Stoica@uhbristol.nhs.uk

Bristol Heart Institute Department of Paediatric Cardiac Surgery, Bristol Royal Hospital for Children, Upper Maudlin Street, Bristol BS2 8BJ Tel +44 117 3428854, Fax +44 1173428850

Conflicts of interest: All authors declare no conflicts of interest relevant to this manuscript.

Funding and ethical: This project was funded by the National Institute for Health Research, Health Services and Delivery Research Program (Project No: 12/5005/06). K. Brown, D. Ridout and V. Tsang were supported by the National Institute for Health Research Biomedical Research Centre at Great Ormond Street Hospital for Children NHS Foundation Trust and University College London. D.M. Dorobantu was supported by a PhD Studentship (grant MR/N0137941/1 for the GW4 BIOMED DTP), awarded to the Universities of Bath, Bristol, Cardiff and Exeter from the Medical Research Council (MRC)/UKRI. The study has ethical approval from London City Road Research Ethics Committee (14-LO-1442). The views expressed are those of the authors and not necessarily those of the National Health Service, National Institute for Health Research, or Department of Health.

Text word count: 3491

Abstract word count: 246 


\section{Glossary of abbreviations}

ASD - atrial septal defect

AVSD - atrioventricular septal defect

CAVSD - complete atrioventricular septal defect

DORV - double outlet right ventricle

HLHS - hypoplastic left heart syndrome

IQR - interquartile range

NCDHA - National Congenital Heart Disease Audit

PA - pulmonary atresia

PAPVC - partially anomalous pulmonary venous connection

PDA - patent arterial duct

PRAiS - Partial Risk Adjustment in Surgery

RV-PA - right ventricle to pulmonary artery

STS - Society of Thoracic Surgeons

TAPVC - totally anomalous pulmonary venous connection

TGA - transposition of great arteries

$\mathrm{uRE}$ - unplanned reintervention

UVH - univentricular heart 
VSD - ventricular septal defect 


\section{Abstract and key words}

Objective: Unplanned reintervention (uRE) is used as an indicator of patient morbidity and quality of care in pediatric cardiac surgery. We investigated associated factors and early mortality after uRE's.

Methods: Morbidity data were prospectively collected in 5 UK centres between 2015 and 2017; uRE included surgical cardiac, interventional transcatheter cardiac, permanent pacemaker and diaphragm plication procedures. Mortality (30-day/6-month) in uRE/no-uRE was reported before and after matching. Predicted 30-day mortality was calculated using the Partial Risk Adjustment in Surgery (PRAiS) score.

Results: A total of 3090 procedures (2861 patients) were included (median age 228 days). There were 146 uRE's, resulting in an uRE rate of 4.7\%. PRAiS score, 30-day mortality and 6-month mortality in uRE and no-uRE groups were: $2.4 \%$ vs $1.3 \%, 8.9 \%$ vs $1 \%$ and $17.1 \%$ vs $2.4 \%$, respectively. After matching, mortality at 6 months remained higher in uRE compared to no-uRE (12.2\% vs $1.4 \%, \mathrm{p}=0.02,74$ pairs). In the uRE group, $21 / 25$ deaths at 6 months occurred when at least one additional postoperative complication was present. In multivariable analysis, neonatal age $(\mathrm{p}=0.002)$, low weight $(\mathrm{p}=0.009)$, univentricular heart $(\mathrm{p}<0.001)$ and arterial shunt $(<0.001)$ were associated with increased risk of uRE, but PRAiS score was not (only in univariable analysis).

Conclusions: Unplanned reinterventions are a relatively frequent complication after pediatric cardiac surgery and are associated with some patient characteristics, but not the PRAiS risk score. Early mortality was higher after an uRE, independent of preoperative factors, but linked to other postoperative complications. 
Key words: unplanned reintervention, reoperation, pediatric cardiac surgery, congenital heart disease, multicentre, mortality 
Central picture legend: Mortality with uRE is higher than without, even after matching by patient characteristics. 


\section{Central message}

Unplanned reinterventions are associated with short term mortality after pediatric cardiac surgery independent of preoperative factors.

\section{Perspective statement}

Morbidities are a quality metric after congenital heart disease, but how they relate to other outcomes is not fully known. This prospective multicentre study shows that unplanned reinterventions, at a rate of $4.7 \%$ and clustered around several conditions, are associated with early mortality; this is independent of preoperative estimated case complexity but not independent of other postoperative complications. 


\section{INTRODUCTION}

Survival after pediatric cardiac surgery have improved in recent decades, registry data showing average early mortality figures of $3 \%$, while the complexity of procedures has increased. ${ }^{1,2}$ Thus, the focus of quality assessment and improvement has shifted towards postoperative morbidity.

The notion of unplanned reintervention ( $\mathrm{URE}$ ) is a promising morbidity measure for quality improvement. Used judiciously, it could identify lapses in patient care, be it incomplete diagnosis, technical issues and residual defects or failed therapeutic plan. ${ }^{3,4}$ On the other hand, uRE's are difficult to measure; we previously investigated early reoperations using UK National Congenital Heart Disease Audit (NCDHA) data, and found it impossible to retrospectively decide if a reintervention is "unplanned". 5 Nevertheless, the National Health Service in the UK has already adopted $\mathrm{URE}$ as a metric of performance, ${ }^{6}$ while a recent NCHDA report highlighted the limitations involved in collecting such data. ${ }^{7}$ The Society of Thoracic Surgeons (STS) database also records uRE's as one of the postoperative morbidities in the US. ${ }^{4,8}$

Another area of debate is whether the increased short-term mortality, either 30 day or in-hospital, observed after unplanned/early reinterventions, ranging from $1 \%^{5}$ to $20 \%{ }^{4}$, is due to the reintervention, patient complexity, unknown factors or a combination of all three. Furthermore, each new post-operative morbidity or event has the potential to trigger a possible future one.

Given the importance of post-operative complications, from patient counselling to resource utilisation, a prospective study was undertaken to evaluate a set of 9 predefined morbidities in 5 UK pediatric cardiac surgery centers for 21 months. ${ }^{9}$ This study aims to undertake a secondary analysis based on this prospectively collected dataset in order to describe the incidence and types of uRE after pediatric cardiac surgery and their relationship with mortality (30 days and 6 months), 
case complexity and other postoperative morbidities. We hypothesised that early mortality is higher in patients undergoing $\mathrm{URE}$ than in those who do not, independent of preoperative patient or procedure factors.

\section{PATIENTS AND METHODS}

\section{Study design and dataset}

Study data collection has been described previously. ${ }^{9}$ Briefly, the dataset included all consecutive patients aged 0-17 years undergoing cardiac surgery (open and closed) or hybrid procedures involving the heart, at 5 participating centers between October 2015 and June 2017. The 5 centres accounted for close to half of the total pediatric cardiac interventions in the country in the 20142017 audit report (https://www.nicor.org.uk/national-cardiac-audit-programme). All clinical decisions were taken by the clinical team at each centre, as per local and national guidelines. The nine morbidities recorded were: unplanned reinterventions, extracorporeal life support, necrotising enterocolitis, prolonged pleural effusion or chylothorax, renal replacement therapy, major adverse events, acute neurological events, feeding issues and post-surgical infection. ${ }^{10}$ Morbidities were attributed locally and discrepancies or complex cases were discussed in monthly team meetings involving study team members from all centres, with decisions being made by consensus. The anonymised cardiac surgical, diagnostic and risk factor data were added to the study dataset from the NCHDA dataset held at each site, which are highly consistent and audited across all centers, with diagnoses, previously described. ${ }^{1,9}$

\section{Definition of reintervention and mortality outcomes}


The study protocol, described previously, ${ }^{9}$ defined uRE as procedures that were not intended during the planning phase, followed an initial cardiac operation, and resulted in "substantive alteration to heart" incorporating cardiac bypass, cardiac non-bypass, permanent pacemaker placement, interventional transcatheter cardiac, and diaphragm plication procedures. The definition excluded mechanical support, other non-cardiac surgery procedures and revisions undertaken within the primary trip to the operating room. In respect of timing, uRE can occur at any time up until 30 days after the index operation, regardless of life status; procedures occurring more than 30 days after the index procedure were considered new entries (index procedures), pertaining to the same patient. The unplanned nature of uRE was judged firstly by the local study team at each center; then queries or complex cases were discussed in meetings involving all study centers. A total of 27 cardiac reinterventions occurring during the 30-day window were part of the planned treatment pathway and were dropped from the dataset.

In order to gather some missing details that had not been included in the initial analysis by Brown et $\mathrm{al}^{9}$ the patient records held at participating centers were re-examined between January and May 2020 for additional data, detailed in Appendix A. Patient survival was ascertained at 30 days and at 6 months after the first index procedure in the dataset, per patient, with $100 \%$ complete followup. A schematic representation of how index procedures, 30 day surgical episodes and mortality events were defined is presented in Appendix A, Supplemental Figure 1.

\section{Risk factor and variable groups}

The clinical data, definitions of terms, and variable classification, such as diagnosis/procedure group for this cohort have been described elsewhere. ${ }^{9}$ 
For the purpose of the current work, the Partial Risk Adjustment in Surgery (PRAiS) score version 2 score was calculated for each index procedure. ${ }^{11}$ The PRAiS score was developed and validated using NCHDA data ${ }^{11}$ and it offers a predicted probability of death at 30 days (expressed as \%) based on several parameters, including diagnosis group, procedure type and comorbidities. In 9 patients where the procedure weight was improbable for age PRAiS was not calculated and age was marked as missing.

\section{Statistical Analysis}

Frequencies are presented as numbers and percentages and all continuous variables as median (inter-quartile range). The Fisher's test was used for comparisons between proportions and the Mann-Whitney U test was used for comparisons between continuous variables.

To identify factors associated with $u R E$, we performed univariable and multivariable competing risks analysis analyses (death is a competing risk to uRE, censoring at 30 days) $)^{12}$ using the following candidate variables: age at procedure (continuous), neonatal age, weight (continuous), low weight for age, patient sex, diagnosis complexity group (ordinal), univentricular heart, PRAiS score (continuous), acquired comorbidity, congenital comorbidity, prematurity, Down's syndrome, severity of illness risk presence, additional cardiac risk factors, procedure type (palliation/corrective/ambiguous), Norwood procedure, arterial shunt, bypass length (none/up to $90 \mathrm{~min} / 90 \mathrm{~min}$ or more) and center. Previous research identified univentricular anatomy and the Norwood and arterial shunt operations as subgroups associated with increased mortality, which is why they were added as separate variables. ${ }^{5}$ A combination of stepwise forward selection and backward elimination was used (model details in Appendix C). The final multivariable model was derived by fitting a logistic regression model for all significant associated factors $(p<0.05)$. 
Variance inflation factor was used to evaluate significant variable collinearity, which was not observed.

To investigate mortality attributable to the presence of $u R E$, cases with an $\mathrm{uRE}$ were matched based on patient and procedure characteristics with controls not having an uRE. This was done using a 1:1 coarsened matching algorithm (CEM command for STATA) with random pruning of unmatched pairs. ${ }^{13}$ The matching was augmented stochastically with an approach similar to that used by Sharabiani et al. previously, ${ }^{14,15}$ with a total of 1000 matching iterations being done. We compared both mortality rates (Fisher test) and time-to-event mortality estimates (log-rank test). Reported mortality at 30 days $/ 6$ months after matching is the median value, while the Fisher/logrank test $\mathrm{p}$ values are the geometrical mean in these 1000 iterations. A detailed description of the matching algorithm can be found in the Appendix C.

All statistical analyses were done using the Stata/SE 16.0 package (StataCorp LLC, College Station, Texas, USA). uRE network graphs (Figures 1,2, Supplemental Figure 2) were drafted using the online Flourish Studio platform (Kiln Enterprises Ltd, London, UK) and then manually edited for readability, using raw numerical data found in Appendix D.

\section{RESULTS}

A total of 3090 surgical 30 day episodes (with index procedures) pertaining to 2861 patients were included, where 2648 patients had one, 197 had two and 16 had three procedures. In the whole cohort, early (30-day) mortality was $1.3 \%$, 6-month mortality was $3 \%$ and the PRAiS score was $1.3 \%$. 
A total of 153 uRE's were reported in $n=146$ patients, resulting in an uRE incidence of $4.7 \%$. Of these, $n=89$ were cardiac surgical $u R E$ (incidence $2.9 \%$ ), $n=24$ cardiac transcatheter (incidence $0.8 \%$ ), $n=26$ pacemaker (incidence $0.8 \%$ ) and $n=14$ diaphragm plication (incidence $0.4 \%$ ). Demographic, clinical and procedural data by uRE presence are detailed in Table 1.

The median time to uRE overall was 10 days (IQR 3-17), this breaks down into 6 (2-15) days for cardiac surgery, 12 (7-19) days for transcatheter procedures, 15 (8-21) days for pacemakers and 14 (11-17) days for diaphragm plications, $(\mathrm{p}=0.002$ for between-group comparison).

\section{Relationship between uRE and short term mortality}

Figure 3 summarises early mortality (30 days and 6 months) in patients with and without uRE, before and after matching, as well as 30 day mortality as predicted by PRAiS score. Mortality at 30 days and 6 months was higher in patients with an uRE than in those without $(8.9 \%$ vs $1 \%$, $\mathrm{p}<0.001$ and $17.1 \%$ vs $2.4 \%, \mathrm{p}<0.001$, respectively) in the whole cohort, as was 30 days PRAiS predicted mortality $(2.4 \%$ vs $1.3 \%, \mathrm{p}<0.001)$. After matching by patient and procedure characteristics, resulting in 74 pairs, 30-day and 6-month mortality were higher in the uRE group compared to controls $(6.8 \%$ vs $0 \%$, Fisher $p=0.1 / \log$-rank $p=0.03$ and $12.2 \%$ vs $1.4 \%$, Fisher $p=$ 0.02/log-rank $p=0.006$, respectively), while PRAiS predicted 30 day mortality was similar $(1.5 \%$ vs $1.4 \%, \mathrm{p}=0.9)$.

\section{Unplanned reinterventions by index procedure type}

Incidence of uRE in specific procedure types ranged from $0-22 \%$ (Table 2). Among the groups of procedures with more than 20 cases, arterial shunts, mitral valve replacements and Norwood operations had most uRE's, with rates of $22 \%, 18.2 \%$ and $13.7 \%$ respectively. 
The relationship between index procedure and reinterventions is complex. Descriptively, we found several procedure groups which were more likely to be linked by uRE's: surgical uRE's were more common after the Fontan pathway procedures and operations involving aortic arch obstructions or ventricular septal defects (VSD)/tetralogy of Fallot (ToF)/complete atrioventricular septal defect (CAVSD) (Figure 1).; transcatheter uRE's were more common after Fontan pathway procedures and arterial shunts (Figure 2A); pacemaker uRE's (Figure 2B ) and diaphragm plications (Appendix D: Supplemental Figure 2) were more common after procedures involving a VSD or right ventricular outflow tract obstruction and those part of the univentricular route. Of note is that death events at 6 months were distributed evenly between main and smaller index procedure nodes. All numerical values pertaining to index-uRE pairs are found in Appendix D.

\section{Factors associated with unplanned reintervention}

Table 3 details factors associated with any uRE, based on univariable and multivariable analysis. Although univariable analysis showed that PRAiS score was associated with uRE risk, the multivariable analysis revealed that only some patient characteristics that go into calculating the PRAiS score remained independently significant (Table 3). There was no center effect in either the univariable or multivariable analyses. Time from index to uRE was not associated with either 30 day or 6 month mortality, overall, or in any of the uRE type subgroups.

\section{Relationship between uRE type, other postoperative morbidities and early mortality}

Postoperatively, patients experiencing an uRE were more likely to also have any of the other specifically collected postoperative morbidities, before or after the uRE (all $\mathrm{p} \leq 0.001$, Table 1 ). 
Of the 146 patients with uRE, 92 also had another postoperative morbidity, while in 54 the only complication was the uRE. As shown in Table 4, the mortality at 30 days and 6 months was significantly higher when uRE was not the only postoperative morbidity, compared to isolated uRE (30-day: $14.1 \%$ vs $0 \%$, 6 -month: $22.8 \%$ vs $7.4 \%$, $<<0.001$ for both comparisons). Early mortality was highest after cardiac surgical uRE's (30-day: 12.4\%, 6-month: 21.4\%) and isolated diaphragm plication uRE (30-day: 1 of 9 patients, 6-month: 3 of 9 patients) and lower after transcatheter cardiac uRE (30-day: 4.4\%, 6-month: 13\%), while no deaths were observed after pacemaker uRE's. Mortality in uRE's with multiple morbidities was consistently higher compared to uRE only, in all three uRE subgroups where deaths were observed (Table 4). Due to the intricacies involved in evaluating sequences of events, a detailed analysis of the interaction between uRE and each morbidity type was not attempted at this time.

\section{DISCUSSION}

Unplanned reinterventions are relatively common after pediatric cardiac surgery, occurring in $4.7 \%$ of procedures in this multicentre prospective study. We hypothesised that uRE's would be associated with early mortality independent of known preoperative factors. We found that mortality was higher at 30 days and 6 months after uRE's compared to those without uRE's (8.9\% vs $1 \%$ and $17.1 \%$ vs $2.4 \%$, respectively) and this difference persisted after matching for preoperative and operative factors (6.8\% vs $0 \%$ and $12.2 \%$ vs $1.4 \%$, respectively, Figure 3 ). uRE's were strongly linked to the occurrence of other morbidities, with fewer early deaths being observed in cases where the only complication was an uRE, compared to multiple morbidities. In terms of uRE types, most of the morbidity and mortality burden was associated with cardiac surgical reinterventions, less so with transcatheter uREs. No deaths occurred in patients having an unplanned pacemaker procedure. 
Our findings underscore the importance of uRE as a quality metric, and more importantly as a clinically relevant outcome, with ramifications for patient counselling given significantly altered patient trajectory. Nevertheless, caution needs to be observed if uRE is to be used as a metric for quality of care, as it does not reflect the entirety of the clinical picture: the different types of postoperative morbidities are clearly interconnected, and linked to later mortality, as previously shown ${ }^{9}$, but the direction of causality among them is not clear. In addition, having uRE alone as a measure of quality could incentivise avoiding or postponing reinterventions, and this in itself could result in poor outcomes.

Few studies describe the reintervention burden in pediatric patients undergoing cardiac surgery. Our previous report from the NCHDA in the UK showed a 3.5\% early cardiac surgical reintervention rate, ${ }^{5}$ while two recent analyses from the STS database found a $5.4 \%$ unplanned reintervention rate (surgical or catheter-based), ${ }^{4}$ and a $2.5 \%$ incidence of transcatheter reinterventions, all very similar figures to the current study, despite very different mechanisms of collection and definitions.

Reinterventions (early or unplanned) have been associated with early mortality in previous reports, and this is true after most types of operations. ${ }^{4,5,8}$ An STS report also found that after adjusting for some patient-related factors, albeit few procedure-related ones, unplanned reintervention was independently associated with operative mortality. ${ }^{4}$ In this study we found that uRE's were more commonly associated with certain procedures, such as those from the Fontan pathway, other palliative operations and operations performed in heart conditions with VSD, aortic coarctation or RVOT obstruction (Figure 1), which is consistent with previous data we reported from the NCHDA. ${ }^{5}$ Neonates, univentricular heart, preoperative severe of illness, and palliative procedures (arterial shunt specifically), were also associated with uRE, and represent patient groups where 
targeted measures to avoid uRE's can be directed, such as more detailed imaging to avoid undiagnosed lesions, or aggressive postoperative surveillance of at risk cases. We found that most deaths occurred after surgical type uRE's, less so after transcatheter uRE. Similarly, Thibault et al. reported that unplanned transcatheter reinterventions increased the mortality risk, but with no correlation to failure to rescue. ${ }^{8}$ We also found a notable burden of mortality after isolated diaphragm plications. While this was intriguing, we were unable to infer on a possible cause, or whether this is simply a spurious result, as most of these patients (7/9) had complex underlying disease or pre-existing comorbidities.

Interestingly, Costello et al. report that the STS-EACTS mortality category, based almost entirely on procedure type, was independently associated with uRE's. ${ }^{4} \mathrm{We}$ found that PRAiS score, also a surgical mortality risk score, was associated with uRE only in univariable analysis, but not multivariable analysis; only some of the characteristics included in the calculation of the PRAiS score were predictive of URE. We speculate that one single preoperative "complexity" score would not be able to accurately predict uRE's, especially one derived from mortality data, as uRE's are also linked to operative performance and postoperative factors. Evaluating the full treatment pathway from preoperative factors to postoperative events is attractive, but conceptually challenging. The recently proposed notion of "residual lesion score" from the Pediatric Heart Network $^{3}$, based on previous work on technical performance scores ${ }^{16}$ is one such attempt, and could offer even more insight into the mechanisms of unplanned reinterventions.

Based on these previous reports, we hypothesised that it is not just patient factors which influence the outcomes after uRE's, but also procedure-related factors. To test this hypothesis, we matched patients by preoperative characteristics and also inside diagnosis and procedure group strata having an $\mathrm{URE}$ remained associated with significantly increased mortality at 6 months, but not at 
30 days, likely due to lack of statistical power for the latter. This supports the logical notion that uRE modifies the outcome pathway predicted by preoperative factors alone. One step further, we performed a post hoc exploratory analysis on how uRE relates to other postoperative complications, and found that most of the deaths occurred in those where more than one complication occurred; the final outcomes in these patients are determined by the interaction of all postoperative events, rather than just one (in this case the uRE). This hypothesis warrants future studies with carefully selected methodologies such as multistate regression models.

The prospective study on which this current work was based was born out of the necessity to better understand the effect of morbidities after pediatric cardiac surgery and to explore routes to improve patient care. This field needed clearer definitions, especially when it comes to cardiac reinterventions. The advantage of a prospective design was that the judgement on whether the reintervention was "unplanned" was taken by consensus, which might make for a more robust classification. Empirically, it was found by the team that in some of the more complex cases, even by combining prospective data collecting with team consensus and re-evaluation, classifying some reinterventions still had a degree of subjectivity. Research staff collecting data needed to be well trained and knowledgeable in the field of congenital heart disease, since many procedure sequences tend to be complex. We advise caution when evaluating if and how "unplanned reintervention" should play a larger role in institutional or national quality of care evaluation. The alternative, "early reintervention" (irrespective of planned /unplanned status) has the disadvantage of indiscriminately including procedures which are part of the natural treatment pathway. ${ }^{5}$ Within the first 30 days this should be a rare occurrence so the clear advantage for early reintervention is that this measure can be extracted with minimal effort from existing databases and subsequently interpreted in context. We believe that the notion of "unplanned reintervention" holds value for 
clinical practice, as shown in the current work, since it can reflect the "true" morbidity the patient suffers. Whether a relatively subjective and complex notion term can be implemented in large scale reporting requires further research. We plan to use the framework provided by this study to compare "unplanned" and "early" reinterventions to understand how they can be better implemented in future quality of care evaluation strategies.

\section{Limitations}

This study included consecutive patients with any type of congenital heart disease, undergoing any type of surgical procedure, and thus resulted in a high degree of patient diversity. The decision on timing and reintervention type was not reviewed by the study team, being assumed that the best decision was taken by the clinical team. This might have resulted in variations in practice, but no centre effects was ultimately observed in relation to the studied outcomes. Classification of a procedure as URE, even based on a pre-stated definition, was subjective at times and required decisions by consensus of the team leading to potential for bias, albeit lessened by the multicenter nature of the study team. The very restrictive matching limited the scope of the analysis post matching to just simple descriptive methods. Although there was robust matching in all available strata limited details on the anatomy, besides diagnosis categories, were available. This can result in unaccounted for differences in matching, although the stochastic approach contributes to minimising this risk. We did not have precise details of the timing for each morbidity occurrence and this limited our interpretation of the relationship between all 9 morbidities and 6-month mortality. Finally, since early reinterventions not considered as uRE were discarded early in the data collection, and detailed information on their nature was not subsequently added to the final dataset, the analysis did not allow for a comparison between the role of uRE and the more conventional early reintervention in predicting outcomes; this is planned to be a future analysis. 


\section{Conclusion}

Unplanned reinterventions are a relatively frequent complication, occurring after $4.7 \%$ of pediatric cardiac operations and are associated with increased early mortality after matching for patient, diagnosis and procedure-related factors. We found that specific, selected factors, rather than the overall estimated risk, or case complexity, were better associated with uRE. Certain diagnoses (univentricular heart), procedures (arterial shunt)or patient profiles (neonate) are independently linked to uRE's, while PRAiS score, as a surgical risk score, is not once several components of its score are independently included. Surgical cardiac uREs were more frequent and had higher mortality compared to transcatheter cardiac uREs, with no deaths observed after pacemaker uREs. Other postoperative complications are more frequent in patients having an $\mathrm{URE}$, and increase early mortality, compared to uRE alone, warranting a more focused look at multimorbidities in this context.

\section{ACKNOWLEDGEMENTS}

We thank all those involved in the initial project design and data collection, which included Alison Jones, Andrew Parry, Anne Keatley-Clarke, Bea Tuten, Bill Gaynor, Branko Mimic, Carrie Cherrington, Isobel Mcleod, Joanne Webb, John Stickley, Jon Smith, Karen Sheehan, Kate Bull, Kate Penny-Thomas, Kathleen Selway, Lisa Allera, Liz Smith, Luke Maidment, Lyvonne Tume, Michael Vath, Natasha Khan, Ray Samson, Rhian Lakhani, Rob Tulloh, Rodney Franklin, Sarah

Bohannon, Sheryl Snowball, Sinead Cummins, Teresa Dickson, Thomas Witter, Tom Treasure, Trevor Ritchens, Vibeke Hjortdal, and all the patients who participated in the study. 


\section{REFERENCES}

1. Brown KL, Crowe S, Franklin R, et al. Trends in 30-day mortality rate and case mix for paediatric cardiac surgery in the UK between 2000 and 2010. Open Hear. 2015;2(1):e000157. doi:10.1136/openhrt-2014-000157

2. Jacobs JP, He X, Mayer JE, et al. Mortality Trends in Pediatric and Congenital Heart Surgery: An Analysis of The Society of Thoracic Surgeons Congenital Heart Surgery Database. In: Annals of Thoracic Surgery. Vol 102. Elsevier USA; 2016:1345-1352. doi:10.1016/j.athoracsur.2016.01.071

3. Nathan M, Trachtenberg FL, Van Rompay MI, et al. The Pediatric Heart Network Residual Lesion Score Study: Design and Objectives. J Thorac Cardiovasc Surg. 2019. doi:10.1016/j.jtcvs.2019.10.146

4. Costello JM, Mongé MC, Hill KD, et al. Associations Between Unplanned Cardiac Reinterventions and Outcomes After Pediatric Cardiac Operations. Ann Thorac Surg. 2018;105(4):1255-1263. doi:10.1016/j.athoracsur.2017.10.050

5. Fiorentino F, Stickley J, Dorobantu D, et al. Early Reoperations in a 5-Year National Cohort of Pediatric Patients With Congenital Heart Disease. Ann Thorac Surg. 2016;101(4):15221529. doi:10.1016/j.athoracsur.2015.12.039

6. Congential Heart Quality Dashboard 2015/16. https://www.england.nhs.uk/commissioning/wp-content/uploads/sites/12/2015/04/congntlhrt-def-15-16.pdf. 
7. National Congenital Heart Disease Audit 2019 Summary Report. https://www.hqip.org.uk/wp-content/uploads/2019/09/Ref-129-Cardiac-NCHDASummary-Report-2019-FINAL.pdf.

8. Thibault D, Wallace AS, Jacobs ML, et al. Postoperative Transcatheter Interventions in Children Undergoing Congenital Heart Surgery: An Analysis of the Society of Thoracic Surgeons Congenital Heart Surgery Database. Circ Cardiovasc Interv. 2019;12(6). doi:10.1161/CIRCINTERVENTIONS.119.007853

9. Brown KL, Ridout D, Pagel C, et al. Incidence and risk factors for important early morbidities associated with pediatric cardiac surgery in a UK population. In: Journal of Thoracic and Cardiovascular Surgery. Vol 158. Mosby Inc.; 2019:1185-1196.e7. doi:10.1016/j.jtcvs.2019.03.139

10. Pagel C, Brown KL, McLeod I, et al. Selection by a panel of clinicians and family representatives of important early morbidities associated with paediatric cardiac surgery suitable for routine monitoring using the nominal group technique and a robust voting process. BMJ Open. 2017;7(5):e014743. doi:10.1136/bmjopen-2016-014743

11. Pagel C, Rogers L, Brown K, et al. Improving risk adjustment in the PRAiS (Partial Risk Adjustment in Surgery) model for mortality after paediatric cardiac surgery and improving public understanding of its use in monitoring outcomes. Heal Serv Deliv Res. 2017;5(23):1164. doi:10.3310/hsdr05230

12. Fine JPJ, Gray RJ, Grey R. A proportional hazards model for the subdistribution of a competing risk. $J \quad A m \quad$ Stat Assoc. 1999;94(446):496-509. 
doi:10.1080/01621459.1999.10474144

13. Blackwell M, Iacus S, King G, Porro G. Cem: Coarsened exact matching in Stata. Stata J. 2009;9(4):524-546. doi:The Stata Journal

14. Sharabiani MTA, Dorobantu DM, Mahani AS, et al. Aortic Valve Replacement and the Ross Operation in Children and Young Adults. J Am Coll Cardiol. 2016;67(24):2858-2870. doi:10.1016/j.jacc.2016.04.021

15. Sharabiani MT, Mahani AS. SAMUR: Stochastic Augmentation of Matched Data Using Restriction Methods. $\mathrm{R}$ package version 0.6. 2015. http://cran.rproject.org/package=SAMUR .

16. Nathan M, Karamichalis J, Liu H, et al. Technical Performance Scores are strongly associated with early mortality, postoperative adverse events, and intensive care unit length of stay-analysis of consecutive discharges for 2 years. J Thorac Cardiovasc Surg. 2014;147(1):389-394, 396.e1-396.e3. doi:10.1016/j.jtcvs.2013.07.044 


\section{FIGURES AND LEGENDS}

Figure 1. Network graph of index-surgical cardiac uRE pairs. All nodes are scaled to the number of procedures, and all connections to the number of specific pairs. Surgical uRE's are more common after Fontan pathway procedures, aortic coarctation/aortic arch hypoplasia interventions and procedures involving a (atrio)ventricular septal defect/tetralogy of Fallot; Complete numerical raw data are detailed in Appendix D.

AoV, aortic valve; ASD, atrial septal defect; AVR, aortic valve replacement; CAV, common atrioventricular valve; CAVSD, complete atrioventricular septal defect; CoA, coarctation of the aorta; MV, mitral valve; DORV, double outlet right ventricle; DKS, Damus-Kaye-Stansel; PA, pulmonary artery; IAA, interrupted aortic arch; PA-VSD, pulmonary atresia with septal defect; PDA, patent arterial duct; RVOTO, right ventricular outflow tract obstruction; RV-PA, right ventricle to pulmonary artery; SAS, subaortic stenosis; SVAS, Supravalvar aortic stenosis repair; SVC, superior vena cava; TAPCV, totally anomalous pulmonary venous connection; ToF, tetralogy of Fallot; TV, tricuspid valve; VSD, ventricular septal defect.

Figure 2. Network graph of index-transcatheter cardiac uRE and index-pacemaker uRE pairs. All nodes are scaled to the number of procedures, and all connections to the number of specific pairs. A) Transcatheter uRE's occur mostly after Fontan pathway procedures and arterial shunts; B) Pacemaker uRE's occur after ventricular septal defect or right ventricular outflow tract obstruction procedures; complete numerical raw data are detailed in Appendix D. Abbreviations in Figure 1 legend.

Figure 3. PRAiS-predicted 30-day mortality, observed 30-day mortality and observed 6- month mortality by uRE presence, in the whole group and in matched pairs. Patients with uRE had higher 
30-day and 6-month mortality than those without and the differences persist even after exact matching. The difference in predicted 30-day mortality between those with and without an uRE is small in the whole group, and not significant after matching, suggesting peri-procedural factors contributing to the mortality effect of the uRE.

PRAiS, Partial Risk Adjustment in Surgery; uRE, unplanned reinterventions. 


\section{TABLES}

Table 1. Demographic, clinical and procedural data in procedures with and without an uRE

\begin{tabular}{|c|c|c|c|c|}
\hline & $\begin{array}{l}\text { With uRE } \\
n=146\end{array}$ & $\begin{array}{l}\text { Without uRE } \\
n=2944\end{array}$ & $\begin{array}{l}\text { Total } \\
n=3090\end{array}$ & $\mathrm{p}$ value \\
\hline \multicolumn{5}{|l|}{ Demographic } \\
\hline Age, (y), (IQR) & $\begin{array}{l}0.3 \\
(0.03-1)\end{array}$ & $\begin{array}{l}0.6 \\
(0.2-3.9)\end{array}$ & $\begin{array}{l}0.7 \\
(0.2-3.8)\end{array}$ & $<0.001$ \\
\hline Male (n, \%) & $82(56.2 \%)$ & $1589(54 \%)$ & $1671(54.1)$ & 0.6 \\
\hline Neonate $(n, \%)$ & $53(36.3)$ & $475(16.1)$ & $528(17.1)$ & $<0.001$ \\
\hline Weight (kg), (IQR)* & $4.7(3.3-7.6)$ & $7.1(4.2-15.2)$ & $6.94(4.1-15)$ & $<0.001$ \\
\hline Low weight for age $(\mathrm{n}, \%) *$ & $55(37.7)$ & $993(33.8)$ & $1048(34)$ & 0.4 \\
\hline \multicolumn{5}{|l|}{ Preoperative clinical } \\
\hline \multicolumn{5}{|l|}{ Diagnosis complexity class } \\
\hline A (most severe/complex) & $23(15.8)$ & $254(8.6)$ & $277(9)$ & \multirow[t]{5}{*}{$<0.001$} \\
\hline $\mathrm{B}$ & $28(19.2)$ & $313(10.6)$ & $341(11)$ & \\
\hline $\mathrm{C}$ & $15(10.3)$ & $310(10.5)$ & $325(10.5)$ & \\
\hline $\mathrm{D}$ & $50(34.2)$ & $973(33.1)$ & $1023(33.1)$ & \\
\hline E (least severe/complex) & $30(20.5)$ & $1094(37.2)$ & $1124(36.4)$ & \\
\hline \multicolumn{5}{|l|}{ Diagnoses with $>50$ patients } \\
\hline VSD & $7(4.8)$ & $328(11.1)$ & $335(10.8)$ & \multirow[t]{4}{*}{$<0.001$} \\
\hline ToF/DORV & $14(9.6)$ & $297(10.1)$ & $311(10.1)$ & \\
\hline CAVSD & $17(11.6)$ & $277(9.4)$ & $294(9.5)$ & \\
\hline $\begin{array}{l}\text { Aortic arch obstruction }+ \\
\text { septal defect }\end{array}$ & $11(7.5)$ & $193(6.6)$ & $204(6.6)$ & \\
\hline
\end{tabular}




\begin{tabular}{|c|c|c|c|c|}
\hline HLHS & $20(13.7)$ & $177(6)$ & $197(6.4)$ & \\
\hline TGA-VSD/DORV-TGA & $14(9.6)$ & $183(6.2)$ & $192(6.2)$ & \\
\hline Functionally UVH & $14(9.6)$ & $173(5.9)$ & $187(6.1)$ & \\
\hline ASD & $0(0)$ & $185(6.3)$ & $185(6)$ & \\
\hline PA-VSD & $14(9.6)$ & $140(4.8)$ & $154(5)$ & \\
\hline $\begin{array}{l}\text { Isolated aortic } \\
\text { stenosis }\end{array}$ & $2(1.4)$ & $141(4.8)$ & $143(4.6)$ & \\
\hline $\begin{array}{ccc}\text { Isolated } & \text { mitral valve } \\
\text { abnormality } & & \end{array}$ & $7(4.8)$ & $73(2.5)$ & $80(2.6)$ & \\
\hline $\begin{array}{l}\text { Ebstein disease and other } \\
\text { tricuspid valve abnormalities }\end{array}$ & $4(2.7)$ & $60(2)$ & $64(2.1)$ & \\
\hline Pulmonary valve stenosis & $2(1.4)$ & $58(2)$ & $60(1.9)$ & \\
\hline Common arterial trunk & $1(0.7)$ & $54(1.8)$ & $55(1.8)$ & \\
\hline \multicolumn{5}{|l|}{ Comorbidities } \\
\hline Acquired comorbidity & $19(13)$ & $437(14.8)$ & $456(14.8)$ & 0.6 \\
\hline Congenital comorbidity & $34(23.3)$ & $682(23.2)$ & $716(23.2)$ & 0.9 \\
\hline Severity of illness risk & $35(24)$ & $339(11.5)$ & $374(12.1)$ & $<0.001$ \\
\hline Down's syndrome & $14(9.6)$ & $263(8.9)$ & $277(8.9)$ & 0.8 \\
\hline $\begin{array}{l}\text { Additional cardiac risk } \\
\text { factors }\end{array}$ & $15(10.3)$ & $215(7.3)$ & $230(7.4)$ & 0.2 \\
\hline Prematurity & $21(14.4)$ & $283(9.6)$ & $304(9.8)$ & 0.06 \\
\hline Any type of UVH & $43(29.5)$ & $371(12.6)$ & $414(13.4)$ & $<0.001$ \\
\hline \multicolumn{5}{|l|}{ Procedural data } \\
\hline \multicolumn{5}{|l|}{ Procedure type (n, \%) } \\
\hline Reparative/corrective & $693(43.2)$ & $1662(56.5)$ & $1724(55.8)$ & 0.001 \\
\hline
\end{tabular}




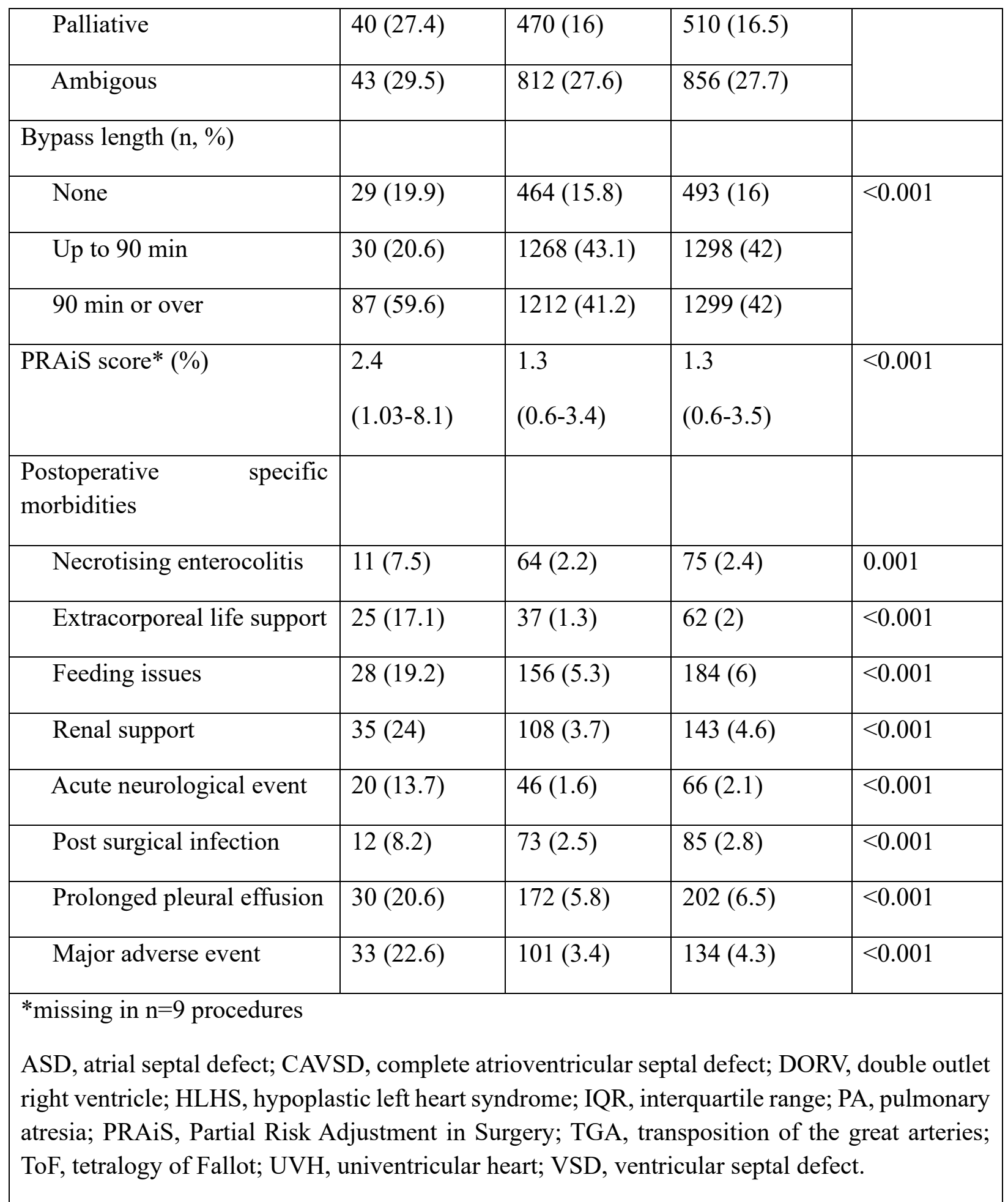




\begin{tabular}{|c|c|c|c|}
\hline & With uRE & Without uRE & Total \\
\hline $\begin{array}{l}\text { Tetralogy and Fallot type DORV } \\
\text { repair }\end{array}$ & $13(6.1)$ & 199 & 212 \\
\hline VSD repair & $9(4.2)$ & 203 & 212 \\
\hline $\begin{array}{ll}\text { Bidirectional cavopulmonary } \\
\text { shunt }\end{array}$ & $8(5.5)$ & 138 & 146 \\
\hline Fontan procedure & $10(7.4)$ & 125 & 135 \\
\hline $\begin{array}{l}\text { Isolated coarctation/hypoplastic } \\
\text { arch repair }\end{array}$ & $9(6.7)$ & 125 & 134 \\
\hline ASD repair & $0(0)$ & 125 & 125 \\
\hline CAVSD repair & $11(9)$ & 111 & 122 \\
\hline Isolated pulmonary artery band & $6(6.2)$ & 91 & 97 \\
\hline Subaortic stenosis repair & $2(2.1)$ & 94 & 96 \\
\hline Vascular ring procedure & $0(0)$ & 87 & 87 \\
\hline Arterial switch (for isolated TGA) & $3(3.5)$ & 82 & 85 \\
\hline Aortic valve repair & $3(4.1)$ & 71 & 74 \\
\hline Norwood procedure & $10(13.7)$ & 63 & 73 \\
\hline $\begin{array}{l}\text { Isolated RV-PA } \\
\text { construction }\end{array}$ & $2(3)$ & 64 & 66 \\
\hline Partial AVSD repair & $0(0)$ & 60 & 60 \\
\hline Pulmonary valve replacement & $0(0)$ & 51 & 51 \\
\hline Arterial shunt & $11(22)$ & 39 & 50 \\
\hline Cardiac conduit replacement & $1(2.1)$ & 47 & 48 \\
\hline Surgical PDA ligation & $3(6.7)$ & 42 & 45 \\
\hline TAPVC repair & $3(7)$ & 40 & 43 \\
\hline Arterial switch + VSD closure & $2(5.6)$ & 34 & 36 \\
\hline
\end{tabular}




\begin{tabular}{|c|l|l|l|l|l|l|}
\hline \multicolumn{5}{|l|}{ Table 3. Factors associated with unplanned reintervention in univariable and multivariable } \\
competing risks analysis \\
\hline
\end{tabular}




\begin{tabular}{|c|c|c|c|c|c|c|}
\hline Up to $90 \mathrm{~min}$ & 0.4 & $0.2-0.7$ & 0.001 & & & \\
\hline 90 min or more & 1.1 & $0.7-1.7$ & 0.7 & & & \\
\hline Norwood operation & 3.2 & $1.6-6.3$ & 0.001 & & & \\
\hline Arterial shunt & 5.6 & $2.9-10.8$ & $<0.001$ & 2.9 & $1.4-5.9$ & 0.003 \\
\hline \multicolumn{7}{|c|}{$\begin{array}{l}\text { Only variables with a } \mathrm{p}<0.1 \text { in univariable analysis are shown. Death was considerec } \\
\text { competing risk to unplanned reintervention. } \\
\text { CI, confidence interval; SHR, subhazard ratio; PRAiS, Partial Risk Adjustment in Surgery. }\end{array}$} \\
\hline
\end{tabular}




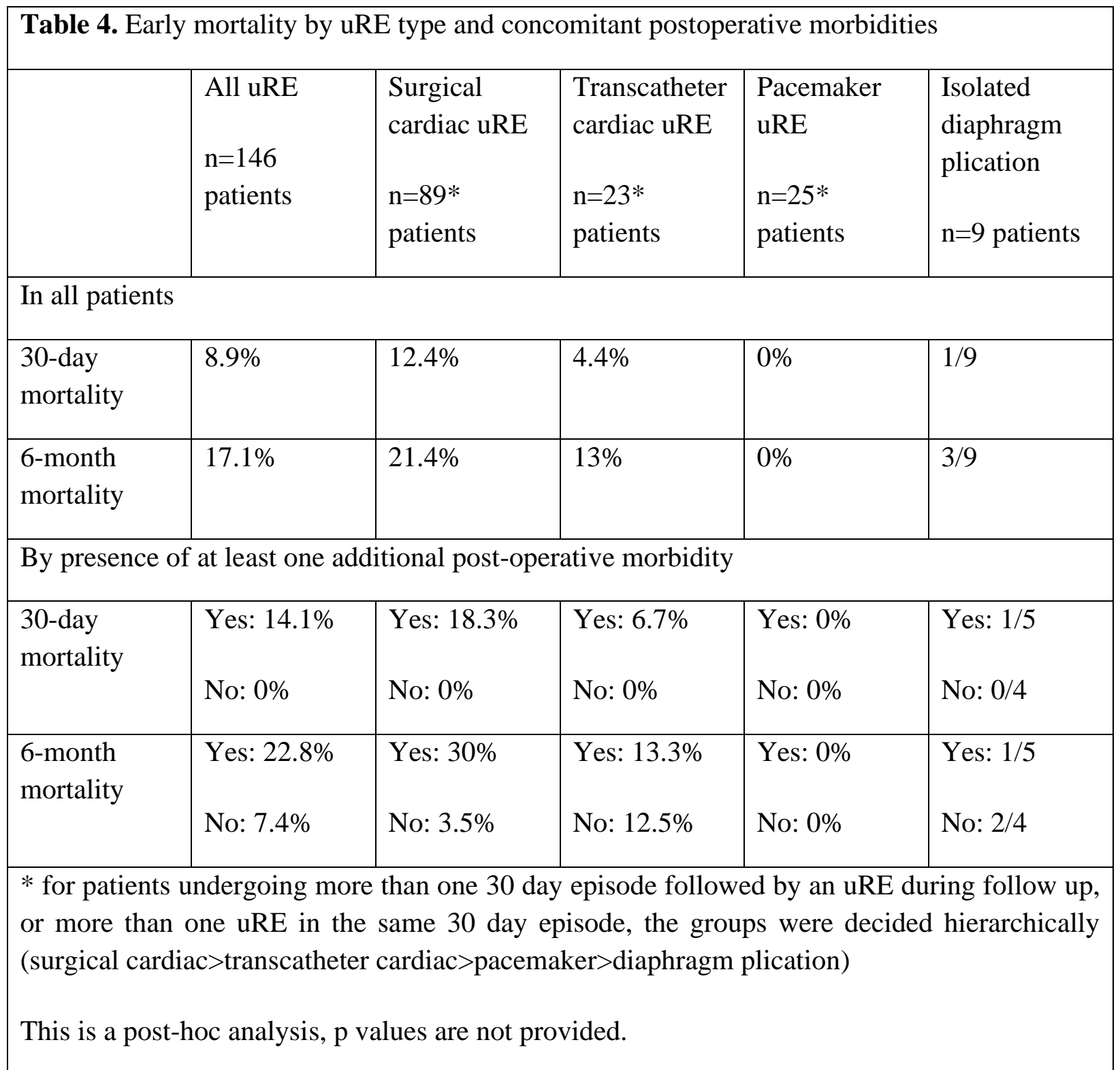

